\title{
Neural substrates of cognitive switching and inhibition in a face processing task
}

\author{
Camille Piguet $^{\mathrm{a}, *}$, Virginie Sterpenich ${ }^{\mathrm{a}}$, Martin Desseilles ${ }^{\mathrm{a}, \mathrm{b}, \mathrm{c}}$, Yann Cojan ${ }^{\mathrm{a}}$, \\ Gilles Bertschy ${ }^{\mathrm{d}}$, Patrik Vuilleumier ${ }^{\mathrm{a}, \mathrm{e}}$ \\ a Department of Neuroscience, Faculty of Medicine, Geneva, Switzerland \\ b Department of Psychiatry, University Hospital of Geneva, Switzerland \\ c Cyclotron Research Centre, University of Liege, Belgium \\ d Department of Psychiatry and Mental Health, Strasbourg University Hospital, University of Strasbourg, INSERMu1114, France \\ e Department of Clinical Neuroscience, University Hospital of Geneva, Switzerland
}

\section{A R T I C L E I N F O}

\section{Article history:}

Accepted 6 June 2013

Available online 14 June 2013

\section{Keywords:}

fMRI

Task-switching

Inhibition

Emotional faces

\begin{abstract}
A B S T R A C T
We frequently need to change our current occupation, an operation requiring additional effortful cognitive demands. Switching from one task to another may involve two distinct processes: inhibition of the previously relevant task-set, and initiation of a new one. Here we tested whether these two processes are underpinned by separate neural substrates, and whether they differ depending on the nature of the task and the emotional content of stimuli. We used functional magnetic resonance imaging in healthy human volunteers who categorize emotional faces according to three different judgment rules (color, gender, or emotional expression). Our paradigm allowed us to separate neural activity associated with inhibition and switching based on the sequence of the tasks required on successive trials. We found that the bilateral medial superior parietal lobule and left intraparietal sulcus showed consistent activation during switching regardless of the task. On the other hand, no common region was activated (or suppressed) as a consequence of inhibition across all tasks. Rather, task-specific effects were observed in brain regions that were more activated when switching to a particular task but less activated after inhibition of the same task. In addition, compared to other conditions, the emotional task elicited a similar switching cost but lower inhibition cost, accompanied by selective decrease in the anterior cingulate cortex when returning to this task shortly after inhibiting it. These results demonstrate that switching relies on domain-general processes mediated by postero-medial parietal areas, engaged across all tasks, but also provide novel evidence that task inhibition produces domain-specific decreases as a function of particular task demands, with only the latter inhibition component being modulated by emotional information.
\end{abstract}

(c) 2013 Elsevier Inc. All rights reserved.

\section{Introduction}

The ability to switch from one task to another is a common cognitive process in everyday life, which is also extensively studied in behavioral research (for a review, see Meiran, 2010; Monsell, 2003). It is well established that such switching induces some behavioral cost (e.g. increased reaction time [RT] and error rate), imputed to changes in executive control operations (e.g. Allport and Wylie, 2000). Changing the current cognitive state requires the disengagement from previous activity and the generation of a new task-set, which as a whole is called "task-set reconfiguration" (Monsell, 2003). Switching costs

\footnotetext{
* Corresponding author at: Laboratory for Neurology and Imaging of Cognition, Neuroscience Department, Faculty of Medicine, University of Geneva, Switzerland, Address: University Medical Center, 1 rue Michel Servet, 1211 Genève 4, Switzerland. Fax: + 41223795402 .

E-mail address: camille.piguet@unige.ch (C. Piguet).
}

may result from both of these reconfiguration processes and the carryover of persisting task-set interference (Kiesel et al., 2010). Several paradigms of task-switching have been designed to differentiate between the inhibition of a first mental set (task) and the reconfiguration necessary to implement a new set (second task) (Arbuthnott, 2008; Hubner et al., 2003; Koch et al., 2004; see, e.g. Mayr and Keele, 2000; Mayr, 2002). This work suggests that, in addition to a simple interruption of the ongoing mental set, some backward inhibition of the preceding task is also necessary during this process, which may act to reduce competition from the previous task demands on the new one. However this process of inhibition is still debated (Kiesel et al., 2010) and its neural substrate remains unresolved.

Evidence for backward inhibition in task-switching is usually obtained by testing sequences of three alternating tasks in $\mathrm{ABA}$ order or in CBA order (for review, see Koch et al., 2010). Indeed, in ABA sequence, switching from $A$ to $B$ requires first to inhibit the ongoing mental processes engaged in $A$, so that returning to A shortly 
afterwards will be less efficient and requires stronger effort. This effect of returning to a previously inhibited task has therefore a measurable behavioral consequence in terms of reaction time when compared to the same task demand in CBA sequences, which also consist of two successive switches, but without returning to a previously inhibited task. Thus, the supplementary cognitive cost for ABA relative to CBA sequences (or "N-2 task-repetition cost") represents a consequence of backward inhibition and leads to slower reaction time, in addition to the cost of switching itself. This inhibition cost shows that a previously inhibited stimulus dimension is still inhibited (or harder to activate) when it becomes again the relevant dimension (Philipp et al., 2008). In contrast, a "pure" cost of switching can be estimated with a different trial sequence (BBA versus AAA), which only involves the interruption of the previous task-set and the generation of a new, non-inhibited task-set. In the present fMRI study, we used a task switching with these 4 different conditions, allowing us to investigate brain systems recruited by the switching processes, as well as those modulated as a consequence of inhibition.

Studies exploring the neural basis of task-switch have implicated various brain regions, including the prefrontal cortex (Dosenbach et al., 2006; e.g. Luks et al., 2002), pre-supplementary motor area (pre-SMA) (e.g. Rushworth et al., 2002; Woodward et al., 2006), parietal regions (e.g. Forstmann et al., 2006), and basal ganglia (Crone et al., 2006; e.g. Ravizza and Ciranni, 2002), all assembled in a distributed network (Brass and von Cramon, 2004; Dove et al., 2000; Dreher et al., 2002; Pollmann et al., 2000). However, the complexity of paradigms used and the multitude of processes supporting task-switching have hampered definite conclusions (Ravizza and Carter, 2008). Inhibition processes associated with switching have more rarely been studied. The inferior frontal gyrus (IFG) is activated during motor response inhibition (Konishi et al., 1998) but also during switching and other cognitive inhibition demands (Derrfuss et al., 2005; Konishi et al., 1999). This brain region might therefore appear as a good candidate for supporting inhibition in task-switching (Aron et al., 2004; Sakai, 2008), but empirical evidence is still very sparse. One behavioral study in a small sample of frontal damaged patients found a dissociation between the right and left hemisphere respectively for inhibition and activation of task-sets (Mayr et al., 2006). Another study using event-related fMRI compared ABA versus CBA task sequences (Dreher and Berman, 2002) with letter stimuli, and reported that the former type of trials produced greater activation in right lateral prefrontal cortex, as well as left inferior temporal cortex and occipital cortex, possibly reflecting the need of "overcoming inhibition". On the other hand, to our knowledge, no neuroimaging study so far has investigated the neural bases of the presumed inhibition component during switching, and there is no direct evidence for a "suppression" of specific task-related representations due to such inhibition when switching from one task to another.

In the current study, we aimed at testing the neural consequences of both inhibition and switching processes during a cognitive task with alternating demands. Despite abundant research in behavioral cognitive psychology, the neural substrates recruited and/or modulated by inhibition during task switching remain poorly known. Whereas inhibition of task-related representations has been hypothesized to account for slower response times in $\mathrm{ABA}$ than $\mathrm{CBA}$ sequences, to our knowledge, there is no empirical evidence to demonstrate that some neural functions are indeed "suppressed" to some degree following the presumed inhibition. Here, using three different judgment tasks with faces, we investigated how previous task inhibition modulated brain activity when returning to the same task and asked whether common or distinctive changes were produced in different task conditions. Thus, we could expect either increased activation in regions previously implicated in cognitive control processes (corresponding to greater "effort" to switch back to the inhibited task-related representations), or reduced activation as a consequence of inhibition (in areas mediating the previously inhibited task-related representations).
In addition, because inhibitory processes might be influenced by emotional signals (Hare et al., 2008; Sagaspe et al., 2011), we also asked whether switching and inhibition would be differentially modulated by emotional information processing. Several studies have reported that emotional cues or emotional states can modulate cognitive control tasks requiring flexibility or shifting abilities (Cohen et al., 2011; Compton et al., 2003; Gray, 2001; Gray et al., 2002; Murphy et al., 2012), suggesting that emotion and executive control may be distinctively connected via brain networks involved in the monitoring of salient information, distribution of attention, and/or selection of appropriate responses (Armony et al., 1997; Compton, 2003). Moreover, the perception of faces and expressions of emotions is an important and particularly efficient ability in humans, guiding social behavior, but also influencing cognitive control and attentional resources in various tasks (Corbetta et al., 2008; Ethofer et al., 2011; Vuilleumier, 2009). Hence, by comparing switching and inhibition in an emotional face condition relative to other non-emotional conditions, our study could also test for neural systems integrating emotion and cognition during task switching, a domain of executive control where affective effects have hitherto not been explored.

For these purposes, we adapted the original paradigm designed by Mayr and Keele (2000) allowing a separate analysis of switching (BBA), inhibition (ABA), and double switch (CBA) conditions, but employing a face categorization task in which three different facial features could be task-relevant (gender, expression, color). We posited that this paradigm should allow us to disentangle the brain substrates recruited during switching (relative to task repetition) and those modulated as a consequence of inhibition (relative to the control condition). First, we predicted that inhibition of task-relevant representations during switching would lead to differential brain activity when returning to the recently inhibited task, as compared with switching to the noninhibited task. Secondly, we hypothesized that, while the brain networks supporting each task should be different and thus lead to different inhibition effects, common regions associated with attention and executive control might be involved as a result of switching and/or inhibition across the three different tasks. Finally, we also expected that the emotional information might produce differential effects on switching and inhibition at both the behavior and brain activation levels (Compton et al., 2003; Sagaspe et al., 2011).

\section{Material and methods}

\section{Participants}

Twenty healthy subjects, recruited by advertising and local database, gave informed written consent before inclusion in the study (10 women and 10 men, mean age 24.9 for both gender, std 5.46). All subjects were right-handed as measured by the Edinburgh handedness inventory (Oldfield, 1971), presented no history of previous psychiatric or neurological diseases, and were not taking any medication. Participants filled the Beck Depression Inventory-II (Beck et al., 1996) and the Beck Anxiety Inventory (Beck et al., 1988) in order to exclude potential psychiatric pathologies. Two subjects were excluded due to high scores on these scales (more than 10 points on the $\mathrm{BDI})$. The remaining 18 subjects were included in the neuroimaging analysis. This study was approved by the ethical committee of the Geneva University Hospital.

\section{Apparatus and stimuli}

A group of three faces (in a triangle configuration) was presented on the screen. These faces were men or women with an emotional expression (happy or sad) and displayed in red or in green color. Faces could be different in three ways, with either different genders, different emotional expressions, or different color hues (Fig. 1). While gender and emotion are relevant dimensions of facial stimuli, we chose 
color as another low-level dimension to match some of the conditions used in the original task (Mayr and Keele, 2000). Participants had to decide which of the three faces was different from the two others along one of these dimensions, depending on the cue presented in the middle of the screen: color, gender, or emotion (Fig. 1). Face photographs were taken from the Karolinska Directed Emotional Faces database (Lundqvist et al., 1998), transformed in black and white, and then tinted with either a red or green color, leading to a total of 96 different stimuli ( 12 identities $* 2$ genders $* 2$ emotions $* 2$ colors). The triangle configuration was always presented at the same localization on the screen, and luminance was controlled among all stimuli. Cues ("color", "gender", or "emotion") were written in white in the middle of the black screen (Fig. 1).

On each trial, a cue appeared $150 \mathrm{~ms}$ before the faces and stayed with their presentation until the subject answered. The display was then followed by a fixation cross of $50 \mathrm{~ms}$. Only if the participants made a mistake, an error feedback screen was presented for $500 \mathrm{~ms}$ with the word "WRONG!!" before the fixation cross. There was no positive feedback, as in the original task of Mayr and Keele (2000). In this paradigm, a rapid succession of trials is necessary to keep task demands high and measure subtle differences in switching costs. To provide some break in this rapid succession of trials, we introduced a brief resting period $(11,000 \mathrm{~ms})$ every 72 trials. The participants had to press the left, middle, or right button if the face that differs from the two others was on the lower left, upper middle, or lower right position, respectively, (using the index, middle, or annular finger). This direct response-mapping set avoids any working memory load and thus more efficiently separates the relevant executive processes from working memory and retrieval.

The paradigm was implemented using E-Prime software 1.0 (Psychology Software Tools Inc., USA) on a standard office PC (Optiplex 755, Dell S.A., Switzerland) running the Windows XP SP3 operating system. Responses were recorded with an MRI-compatible response button box $(\mathrm{HH}-1 \times 4-\mathrm{CR}$, Current Designs Inc., USA).

\section{Design}

Our paradigm was directly adapted from classic behavioral studies of task switching that used other visual stimuli (Arbuthnott, 2008; Lien et al., 2006; Mayr, 2002; Mayr and Keele, 2000). We created 6 lists of 72 trials, with a pseudorandom sequence. For each trial, we considered the two previous trials to determine the condition in which this trial was assigned. For example, in a sequence colorgender-color, the second color task was used for assessing the effect of inhibition ( $=\mathrm{ABA}$ ). Sequences were built so that any given trial $\mathrm{N}$ belonged to one of the four experimental conditions. For each list of 72 trials, we made sure to have a mean of 10 trials for the condition "inhibition" ( $\mathrm{N}-1$ different and $\mathrm{N}-2$ same, e.g. $\mathrm{ABA}$ ), 10 trials for the condition "double switch" ( $\mathrm{N}-1$ and $\mathrm{N}-2$ different, e.g. CBA), and 20 for the pure "switch" condition ( same N-1 and N-2 followed by a switch, e.g. BBA). The rest of the trials were repetition trials (three consecutive trials with the same task, e.g. AAA). Each of the three tasks occurred 24 times per list. The faces were randomly distributed but the identity, color, gender, and emotion were counterbalanced between lists. We made sure that they were no direct repetition of faces. Each participant performed 4 fMRI runs, each with 3 distinct trial lists, presented in a random order counterbalanced between subjects, for a total of 864 trials per subject. Each participant practiced one supplemental run before entering the scanner.

\section{Data acquisition}

Functional MRI data were acquired with a 3T scanner (Trio TIM, Siemens) using a gradient echo-planar (EPI) sequence in a rapid event-related protocol [35 transverse slices with 20\% gap, voxel size: $3 \times 3 \times 3.6 \mathrm{~mm}$, repetition time (TR): $2040 \mathrm{~ms}$, echo time (TE): $30 \mathrm{~ms}$, flip angle (FA): $80^{\circ}$, field of view (FOV): $192 \mathrm{~mm}$ ]. Between 193 and 318 scans (mean of 260) were acquired for each session of the task, depending on how fast the subject answered. A structural scan was acquired at the end of the fMRI session [T1-weighted 3D MP-RAGE sequence, TR: $1900 \mathrm{~ms}$, TE: $2.32 \mathrm{~ms}$, TI: $900 \mathrm{~ms}$, FA: 9, FOV: $230 \mathrm{~mm}$, matrix size $256 \times 256 \times 192$, voxel size: $0.898 \times 0.898 \times 0.9 \mathrm{~mm}$ ]. Stimuli were displayed using an LCD projector (CP-SX1350, Hitachi, Japan) on a screen positioned at the rear of the scanner, which the participants could comfortably see through a mirror mounted on the standard 12 channel head-coil.

\section{Data analysis}

Statistical analyses of behavioral data were conducted using SPSS software (IBM) version 17 and 19. Conditions were compared using a factorial design with 4 conditions (Switch, Repeat, Inhibition, Double switch) and 3 tasks (Color, Gender, Emotion).

fMRI data were preprocessed and analyzed with SPM5 (http:// www.fil.ion.ucl.ac.uk) implemented in Matlab (R2007b Mathworks). Functional scans were first realigned using iterative rigid body transformations that minimize the residual sum of square between the first and subsequent images. They were then normalized to the MNI EPI template (2D spline, voxel size: $2 \times 2 \times 2 \mathrm{~mm}$ ) and finally spatially smoothed with a Gaussian kernel with full-width at half maximum (FWHM) of $8 \mathrm{~mm}$. The high-resolution structural image was co-registered and normalized with the mean image of the EPI series.

Data were processed using a two-step analysis, taking into account the intraindividual and interindividual variance. For each participant,

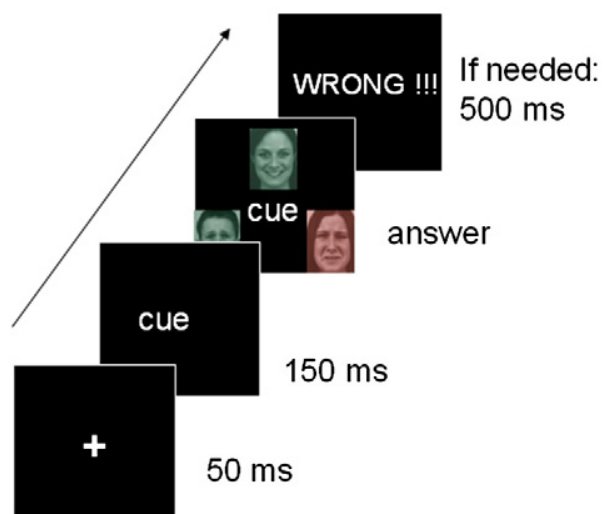

Answer:

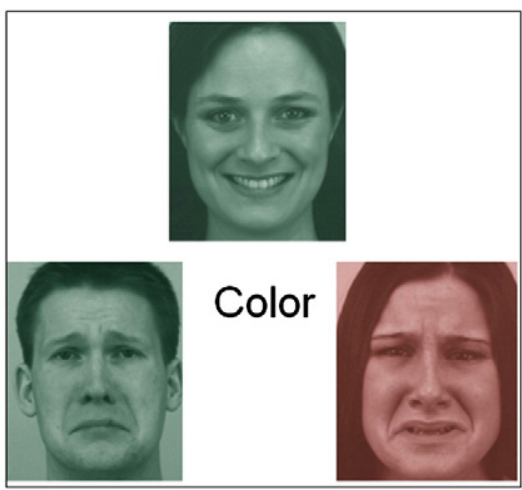

2

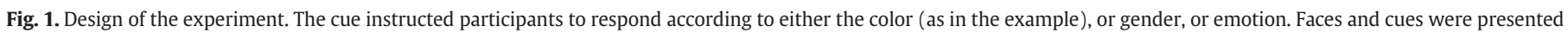
in a pseudo-random order, so that task demands and switching were unpredictable for the subject. 
brain responses were modeled at each voxel, using a general linear model (GLM). There were 12 conditions modeled for the task: Switch_Emotion, Switch_Color, Switch_Gender, Repeat_Emotion, Repeat_Color, Repeat_Gender, Inhibition_Emotion, Inhibition_Color, Inhibition_Gender, DoubleSwitch_Emotion, DoubleSwitch_Color, and DoubleSwitch_Gender, depending on the task and the condition. The onsets were placed at the beginning of the presentation of the faces, taking into account the two previous trials to determine the condition. The trials where subjects did not respond correctly as well as the two following trials were discarded as an additional "wrong" condition to avoid contamination by error effects. We also discarded trials with response times longer than $3000 \mathrm{~ms}$, plus again the two following trials. This finally resulted in the inclusion of a similar proportion (78-82\%) of trials across all experimental conditions.

The ensuing onset vectors were convolved with the canonical hemodynamic response function (HRF) and used as regressors in the individual design matrix. Movement parameters estimated during realignment (translations in $\mathrm{x}, \mathrm{y}$, and $\mathrm{z}$ directions and rotations around $\mathrm{x}-, \mathrm{y}-$, and z-axes) and a constant were also included as a variable of no interest. A high-pass filter was implemented using a cut-off period of $128 \mathrm{~s}$ to remove the low-frequency drifts from the time-series. Serial autocorrelations were estimated with a restricted maximum likelihood algorithm using an autoregressive model of order 1.

The individual summary statistical images were then used in a second-level analysis, corresponding to a random-effect analysis. This analysis was conducted on contrast images of each condition from each subject, using ANOVA flexible factorial design at this second level. As standard practice, activations were considered as significant at a voxel level of $\mathrm{p}<0.001$ (uncorrected, whole brain analysis) with a cluster threshold of $>5$ voxels, unless reported otherwise. Coordinates were reported using the MNI (Montreal Neurological Institute) template. The main effects of different trial conditions (switching, inhibition) as well as the interaction effects between conditions (Switch, Repeat, Inhibition, DoubleSwitch) and Task (Emotion, Color, Gender) were estimated by linear contrasts between corresponding activation maps at the second level. Due to the high trial frequency and event-related design used in our study, plus the constant recruitment of executive control and switch processes on all successive trials, we expect relatively small BOLD fluctuations but highly selective. Using a combination of permissive intensity at the voxel level and a cluster size threshold is a reliable procedure to produce a desirable balance between Types I and II error rates in these conditions (Lieberman and Cunningham, 2009).

\section{Results}

\section{Behavioral results}

The accuracy was overall good with an average of $94.6 \%$ ( std $=4.3$ ) of correct responses (Table 1 ). The $4 \times 3$ repeated measure ANOVA (condition $\times$ task) on accuracy across trial types revealed only a main effect of task $(F(2,16)=14.557, \mathrm{p}<0.001)$. Pairwise comparisons showed that the participants performed better for the Color task compared to Emotion $(p<0.001)$ and Gender $(p=0.001)$ tasks, and better for the Repeat condition compared to the Inhibition condition $(p=0.038)$ and the DoubleSwitch condition ( $p=0.046$ ). Reaction times (RT) were computed for each trial type and each task (Table 1) and also showed the expected effects for conditions of switch. When performing a $4 \times 3$ repeated measure ANOVA (condition $\times$ task), we observed a significant main effect of condition $(F(3,15)=11.006, p<0.001$, Fig. $2 A)$. We also found a main effect of task $(F(2,16)=141.65, p<0.001)$, with shorter response times in the Color task than both the Gender $(\mathrm{p}<0.001)$ and Emotion $(\mathrm{p}<0.001)$ tasks, with no difference between the latter two (Fig. 2B). The interaction between task and condition was not significant. When comparing the experimental conditions of interest, there was a significant slowing on Switch v. Repeat trials ( $p<0.001$ ), consistent with
Table 1

Reaction times in ms and accuracy in percentage.

\begin{tabular}{llll}
\hline Mean RT $(\mathrm{std})$ & Emotion & \multicolumn{1}{l}{ Color } & Gender \\
\hline Switch & $1433.5(55.1)$ & $725(29.5)$ & $1448.7(46)$ \\
Repeat & $1343.4(40.6)$ & $659(20.1)$ & $1402.5(37.9)$ \\
Inhibition & $1433.4(57.4)$ & $767.5(49.3)$ & $1476.4(80)$ \\
DoubleSwitch & $1416.8(76.4)$ & $751.3(40.6)$ & $1428.1(67.5)$ \\
Switch cost & $90.15(121.9)$ & $65.99(54.04)$ & $46.29(68.31)$ \\
Inhibition cost & $16.66(95.75)$ & $16.21(60.22)$ & $48.32(99.59)$ \\
\% Accuracy & Emotion & Color & Gender \\
\hline Switch & 92.20 & 98.55 & 92.05 \\
Repeat & 93.22 & 99.27 & 92.93 \\
Inhibition & 91.35 & 98.03 & 93.35 \\
DoubleSwitch & 92.28 & 98.48 & 91.16 \\
\hline
\end{tabular}

the predicted switching cost. Likewise, Inhibition trials were significantly slower than DoubleSwitch trials $(\mathrm{p}<0.05$ ), demonstrating a reliable N-2 task repetition effect overall.

Both the switching and inhibition costs were present in each of the three tasks, albeit with a large variability (see Table 1 ). Because we were particularly interested in comparing inhibition and switching process for different task demands, more specific comparisons were performed for each task separately, despite the absence of significant task $\times$ condition interaction in the global ANOVA above. As there was a main difference in absolute RTs between tasks, we first computed the differential value for the switch cost (RT condition switch - RT condition repeat) and the inhibition cost (RT condition inhibition RT condition double switch), separately for each task. We then tested for the reliability of these costs across the participants using Wilcoxon signed test in order to verify whether they were significantly greater than zero in each task condition. Results showed a reliable positive effect for switch costs in all three tasks ( $p \leq 0.01$ in all cases, one-tailed) and a reliable effect for inhibition costs in the color and gender tasks ( $\mathrm{p} \leq 0.055)$ but not the emotion task $(\mathrm{p}=0.25)$.

Taken together, these behavioral data indicate that our paradigm produced significant switch and inhibition costs overall, in accordance with previous studies of task switching using similar trial sequences (De Lissnyder et al., 2010; Mayr and Keele, 2000; Whitmer and Banich, 2007). However, while the switch cost was consistently present and positive in all three tasks, the inhibition cost appeared less consistent in the emotion task than in the other two tasks. This difference must be taken with caution, however, as the average cost values were numerically comparable across conditions, and no significant difference was identified in the main ANOVA.

\section{fMRI results}

\section{Main effects of switch conditions}

We first examined the effect of switching, contrasting the Switch trials with the Repeat trials (BBA v. AAA). This revealed activations in bilateral medial superior parietal lobule (SPL), as well as in the left intraparietal sulcus (IPS), posterior cingulate cortex (PCC), and extrastriate visual cortex (Fig. 3, Table 2). Likewise, when contrasting the three conditions with a switching component (switch, double switch, and inhibition) against the repetition condition (Switch + Inhibition + DoubleSwitch $>$ Repeat trials), we observed similar activations in the SPL and PCC, with additional effects in anterior cingulate cortex (ACC). The parameter estimates of activity (beta values) extracted from the left SPL revealed that this region was not only more activated in all switching trials relative to repeated trials (Fig. 3 ), but also less activated in the inhibition condition relative to both the DoubleSwitch and Switch conditions ( $p<0.05$ in post hoc t-tests). This pattern is consistent with a facilitation role in switching, which becomes less efficient after inhibition.

The opposite contrast (Repeat $>$ Switch) showed activation in right supramarginal gyrus, bilateral superior frontal gyrus, and left 


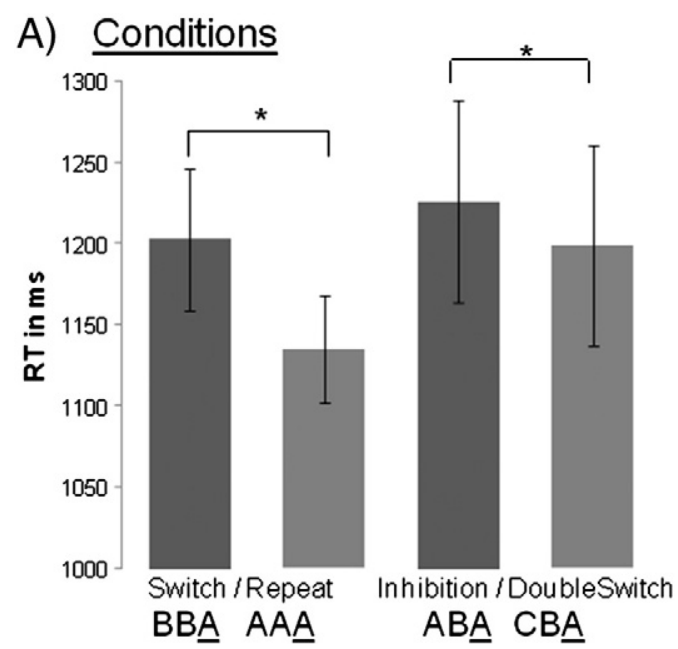

\section{B) $\underline{\text { Tasks }}$}

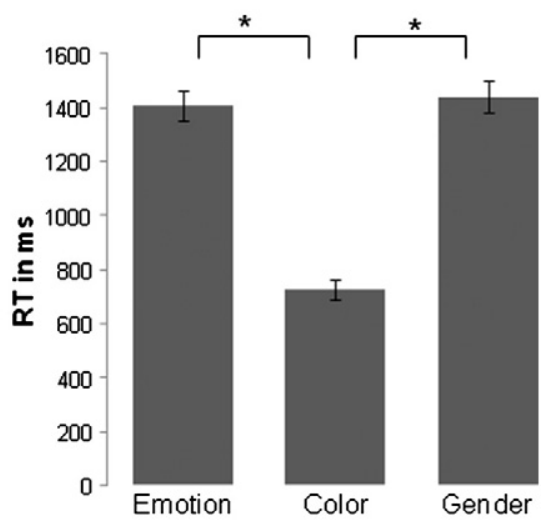

Fig. 2. Behavioral data. A. Absolute response times by condition (mean $+\mathrm{std}$ ). B. Absolute response times by task (mean + std).

caudate nucleus (Table 2). These effects could reflect the fact that repetition trials involved a form of "intradimensional shift" (see Robbins, 2007), distinct from task switching, since there was no change of the task rule but a change in stimuli on those trials.

We then specifically tested for the effect of inhibition by comparing the $\mathrm{N}-2$ repetition trials (ABA) to their DoubleSwitch condition (BCA). The contrast (Inhibition $>$ DoubleSwitch) did not show any significant activation of interest, which is not surprising given that no extra inhibitory process was expected to take place on these trials (i.e. inhibition per se occurred in the preceding $\mathrm{N}-2$ trial). The opposite contrast (DoubleSwitch > Inhibition) highlighted a similar network as found for the Switch v. Repeat trials, with activation peaks in left medial SPL and PCC (Table 2), which confirms that switching processes may be less efficiently recruited as a consequence of the previous inhibition on the $\mathrm{N}-2$ trials. This global decrease in SPL is consistent with the overall RT cost observed at the behavioral level on Inhibition trials.

\section{Interactions with task}

The interaction between trial conditions (Switch, Repeat, Inhibition, DoubleSwitch) and task demands (Emotion, Color, Gender) was examined for the switching and inhibition effects separately. These analyses revealed that switching to a new task produced distinctive increases
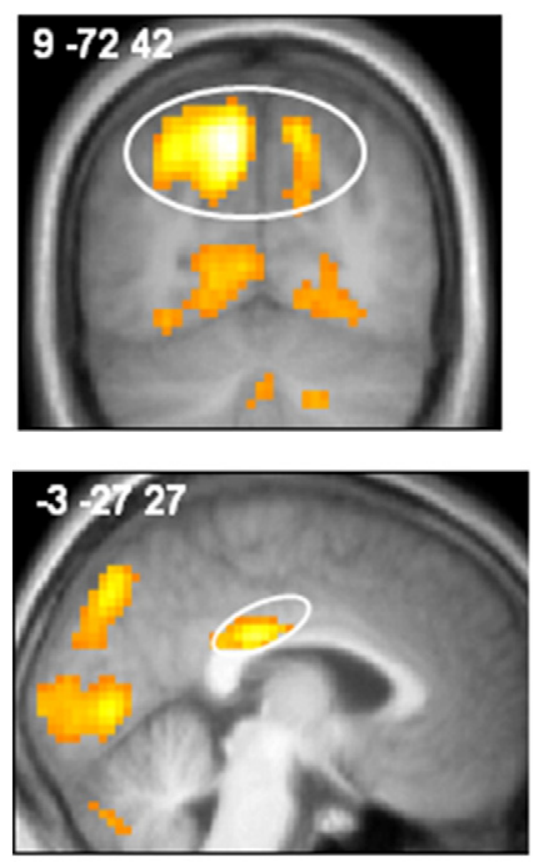

switch

\section{repeat $\square$ inhibition}

doubleswitch

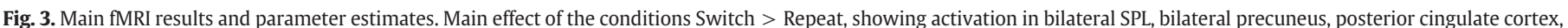

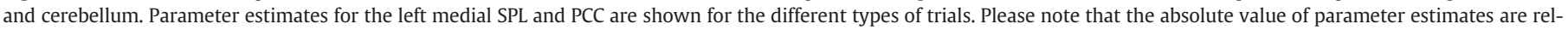

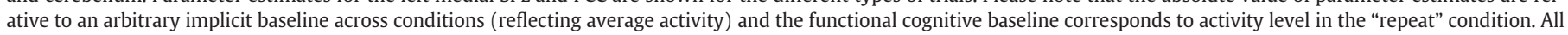
regions are displayed on the mean structural image at $\mathrm{p}<0.001$ uncorrected. ${ }^{*}$ significant at $\mathrm{p}<0.05$. 
Table 2

Regions activated by the different trial types ${ }^{*} \mathrm{p}<0.001$ unc., cluster size threshold 5 vox.

\begin{tabular}{lrllll}
\hline Anatomical label & $\mathrm{x}$ & $\mathrm{y}$ & $\mathrm{z}$ & Voxels & Z-score* $^{*}$ \\
\hline Switch > Repeat & & & & & \\
Left medial SPL & -9 & -72 & 42 & 2016 & 6.51 \\
Left IPS & -30 & -51 & 39 & Above & 4.22 \\
Left PCC & -3 & -27 & 27 & 114 & 5.23 \\
Right fusiform gyrus & 39 & -51 & -24 & 98 & 4.23 \\
Left inferior occipital cortex & -51 & -60 & -15 & 157 & 4.16 \\
Repeat > Switch & & & & & \\
Right inferior frontal gyrus & 45 & 39 & -6 & 8 & 3.41 \\
Left superior frontal gyrus & -12 & 54 & 27 & 20 & 3.92 \\
Right dorsomedial frontal cortex & 6 & 45 & 36 & 13 & 3.73 \\
Left caudate nucleus & -12 & 15 & 12 & 10 & 3.51 \\
Right supramarginal gyrus & 60 & -24 & 30 & 68 & 4.2 \\
Inhibition > DoubleSwitch & & & & & \\
No significant activation & & & & & \\
DoubleSwitch > Inhibition & & & & & \\
Left medial SPL & -6 & -72 & 39 & 41 & 4 \\
Left PCC & -15 & -45 & 30 & 14 & 3.77 \\
\hline
\end{tabular}

in brain regions involved in the specific processing demands of the new task. The interaction contrast (Switch $>$ Repeat) $\times($ Emotion $>$ others tasks) showed activations in the right insula and right striatum (although at a slightly lower threshold, $\mathrm{p}<0.005$, Table 3, Fig. 4A), two regions associated with affective processes. The contrast (Switch $>$ Repeat $) \times($ Color $>$ others tasks) showed activation in bilateral posterior fusiform gyrus and right occipital cortex (Table 3 , Fig. 4C), two visual areas usually recruited during color perception. Finally, the contrast (Switch $>$ Repeat) $\times$ (Gender $>$ other tasks) showed increases in bilateral prefrontal areas and basal ganglia (Table 3, Fig. 4E).

Conversely, we then also tested whether different regions would be less activated as a result of inhibition in the different task conditions, by comparing Inhibition versus DoubleSwitch trials for each task relative to the others. These contrasts showed activations in the insula bilaterally and left superior temporal sulcus (STS) for the interaction (DoubleSwitch $>$ Inhibition) $\times($ Emotion $>$ others tasks); in occipital visual areas for the interaction (DoubleSwitch $>$ Inhibition) $\times$ (Color $>$ other tasks); and in the right basal ganglia for the interaction (DoubleSwitch $>$ Inhibition $) \times($ Gender $>$ others tasks) (Table 3$)$. These data suggest that inhibition on $\mathrm{N}-2$ trials led to less effective recruitment of these task-specific regions when subsequently returning to the same task on trial $\mathrm{N}$.

These effects and the parameter estimates of activity extracted from the corresponding brain areas are illustrated in Fig. 4. It is worth noting that for each task separately, the regions showing greater increases during switching also showed a greater decrease for inhibition. This was the case for the right insula in the emotion task (Fig. 4A and B), the visual cortex (and postcentral gyrus) in the color task (Fig. 4C and D), and basal ganglia for the gender task (Fig. 4E and F). This similarity between the two interaction effects is all the more striking since they were obtained by comparing totally different trials (Switch $>$ Repeat and DoubleSwitch > Inhibition, respectively).

\section{Interaction with emotion}

Given the notion that emotional processing may exert distinct modulatory effects on executive control and response inhibition mechanisms (Compton, 2003; Hare et al., 2008; Murphy et al., 2012; Sagaspe et al., 2011), we also examined whether the inhibitory component contributing to task switching would differ in the emotion condition relative to the other tasks. Behaviorally, in support of this notion, we found that the emotion task was associated with less robust backward inhibition, with the magnitude of RT costs being more variable and non-significantly greater than zero across participants, unlike other conditions (cf. above). We therefore tested
Table 3

Regions activated by the interaction between trial condition and task ${ }^{*} \mathrm{p}<0.001$ unc., cluster size threshold 5 vox, except ${ }^{* *} \mathrm{p}<0.005$ unc.

\begin{tabular}{|c|c|c|c|c|c|}
\hline Anatomical label & $\mathrm{x}$ & $\mathrm{y}$ & $\mathrm{z}$ & Nbr vox & Z-score* \\
\hline \multicolumn{6}{|l|}{ (Switch $>$ Repeat $)>($ Color $>$ others $)$} \\
\hline Left postcentral gyrus & -51 & -21 & 45 & 305 & 5.53 \\
\hline Left postcentral gyrus & -57 & -18 & 21 & 72 & 4.47 \\
\hline Right inferior fusiform cortex & 36 & -75 & -3 & 125 & 4.84 \\
\hline Left posterior fusiform cortex & -45 & -60 & -9 & 189 & 4.18 \\
\hline Right occipital cortex & 12 & -93 & 6 & 178 & 4.26 \\
\hline \multicolumn{6}{|l|}{ (Switch $>$ Repeat $)>($ Gender $>$ others $)$} \\
\hline Right middle frontal gyrus & 33 & 15 & 48 & 72 & 4.19 \\
\hline Left middle frontal gyrus & -30 & 18 & 51 & 31 & 3.6 \\
\hline Right superior frontal gyrus & 9 & 30 & 48 & 86 & 4.12 \\
\hline Left superior frontal gyrus & -12 & 48 & 30 & 21 & 3.83 \\
\hline Right caudate nucleus & 15 & 15 & -3 & 53 & 3.97 \\
\hline Right striatum (putamen) & 24 & 9 & -12 & Above & 3.69 \\
\hline Left medial SPL & -6 & -45 & 63 & 12 & 3.6 \\
\hline \multicolumn{6}{|l|}{$($ Switch $>$ Repeat $)>(\text { Emo }>\text { others })^{* *}$} \\
\hline Right inferior frontal gyrus & 45 & 36 & -6 & 17 & 3.03 \\
\hline Right insula & 45 & 12 & -12 & 21 & 3.05 \\
\hline Right striatum (pallidum) & 24 & -3 & -3 & 35 & 3.28 \\
\hline Right striatum (putamen) & 21 & 6 & 0 & Above & 3.08 \\
\hline \multicolumn{6}{|c|}{ (DoubleSwitch $>$ Inhibition $)>($ Color $>$ others $)$} \\
\hline Left postcentral gyrus & -45 & -24 & 63 & 53 & 3.88 \\
\hline Right posterior occipital cortex & 12 & -93 & 6 & 22 & 3.59 \\
\hline Left middle occipital cortex & -33 & -87 & 15 & 8 & 3.43 \\
\hline Left superior occipital cortex & -15 & -93 & 6 & 15 & 3.41 \\
\hline \multicolumn{6}{|c|}{ (DoubleSwitch $>$ Inhibition $)>($ Gender $>$ others $)$} \\
\hline Right striatum (putamen) & 27 & 12 & -3 & 9 & 3.55 \\
\hline \multicolumn{6}{|c|}{ (DoubleSwitch $>$ Inhibition $)>($ Emo $>$ others $)$} \\
\hline Left insula & -27 & 9 & -15 & 56 & 4.22 \\
\hline Right insula & 36 & -6 & -6 & 17 & 3.73 \\
\hline Right hippocampus & 24 & -33 & -9 & 34 & 4.08 \\
\hline Left middle temporal gyrus & -48 & -24 & -9 & 42 & 4.09 \\
\hline Right superior temporal gyrus & 54 & -15 & -6 & 40 & 3.96 \\
\hline Left superior temporal sulcus (STS) & -63 & -51 & -6 & 46 & 4.23 \\
\hline Right postcentral gyrus & 42 & -12 & 36 & 15 & 4.02 \\
\hline
\end{tabular}

for any difference in the Inhibition condition that arose during the Emotion task but not during the other two tasks (Gender or Color). First, we computed the contrast (DoubleSwitch v. Inhibition) for the Emotion condition only, and then applied an exclusive masking procedure with similar contrasts (DoubleSwitch v. Inhibition) from the Color or Gender conditions (or both combined). This analysis revealed a significant decrease in anterior cingulate cortex (ACC; $\mathrm{x}, \mathrm{y}$, $\mathrm{z}=-15,2424 ; \mathrm{Z}$-score $=3.78$ ) for inhibition trials, in addition to the emotion-specific decreases in insula and striatum as identified in the task $\times$ condition interaction analyses above (see preceding section).

Finally, to confirm that this decrease in ACC was selective to Inhibition trials and not observed in other switching conditions, we also performed an interaction analysis in which we compared conditions involving switching only with conditions involving switching plus the consequence of inhibition, i.e., (DoubleSwitch $>$ Inhibition $) \times$ (Repeat $>$ Switch) for the emotional task relative to the other tasks (Emotion $>$ Sex + Color). This analysis once again found a significant effect in ACC ( $x, y, z=-6$ 21 33; Z-score = 3.38, Fig. 5), which confirms a distinct modulation of the inhibition cost in this region during the emotion task. These results indicate that the ACC might be less strongly recruited when returning to the emotion task on trial $\mathrm{N}$ due to less efficient inhibition of this task set in the preceding $\mathrm{N}-2$ trials. No such effects were observed for the other tasks.

\section{Discussion}

We used event-related fMRI to investigate the neural substrates of two distinct cognitive processes associated with task-switching, namely the effect of inhibition of a previous task-set and the generation of a new task-set. Our behavioral data revealed the predicted 
A) $(\mathrm{S}>\mathrm{R})>($ Emotion $>$ others $)$ : right insula
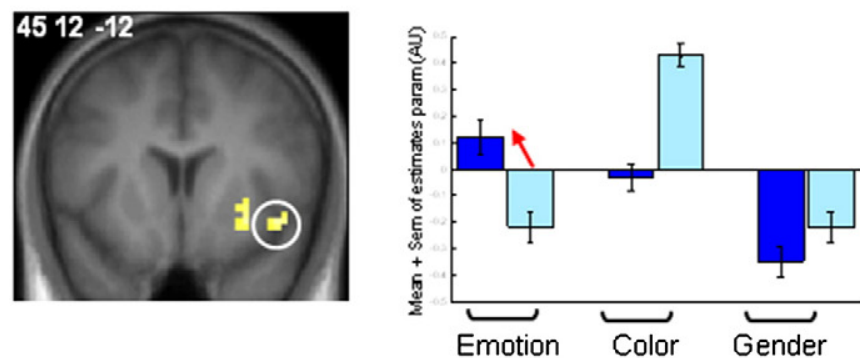

C) $(\mathrm{S}>\mathrm{R})>($ Color $>$ others $)$ : right calcarine cortex
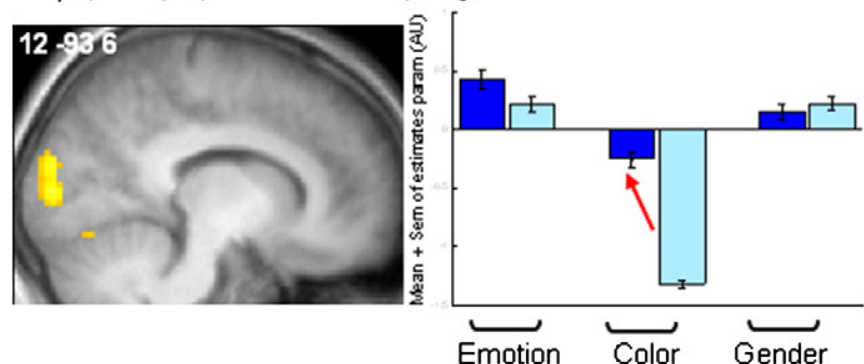

E) $(S>R)>($ Gender $>$ others): right striatum
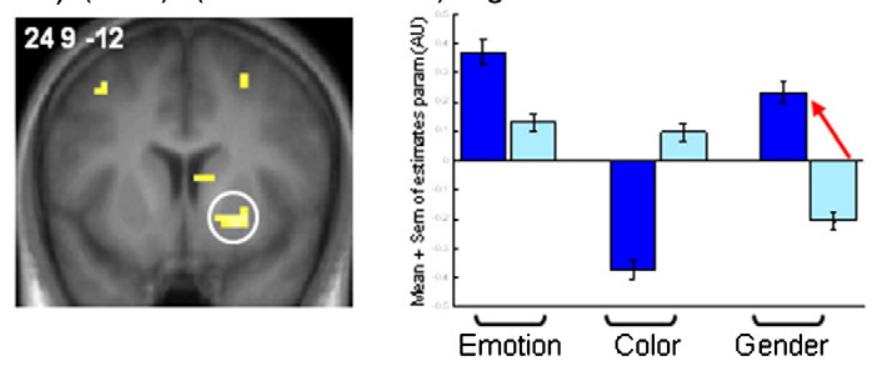

switch repeat
B) $(\mathrm{I}<\mathrm{C})>($ Emotion $>$ others $)$
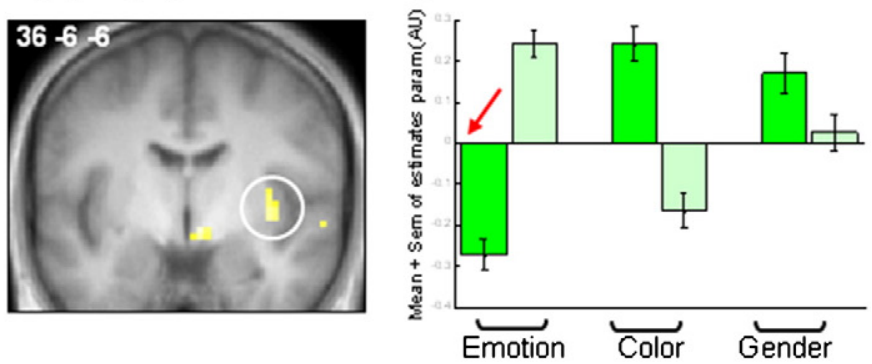

D) $(\mathrm{I}<\mathrm{C})>$ (Color $>$ others)
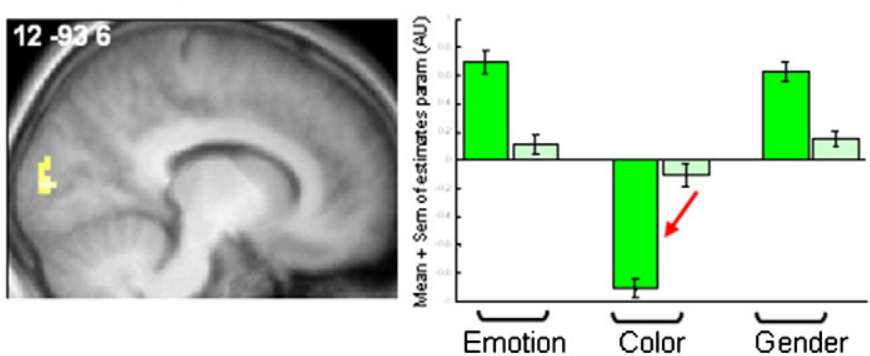

F) $(1<\mathrm{C})>($ Gender $>$ others $)$
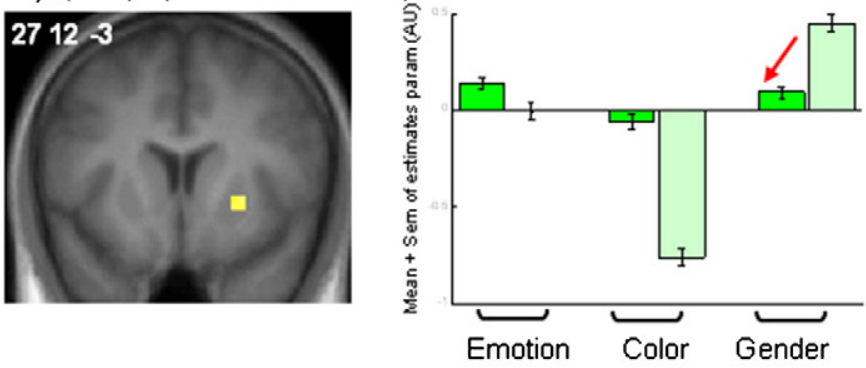

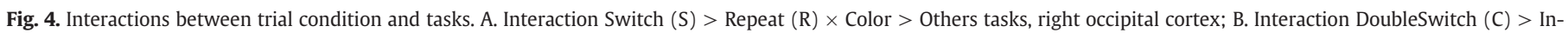

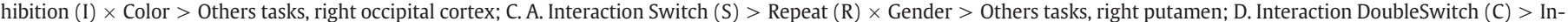

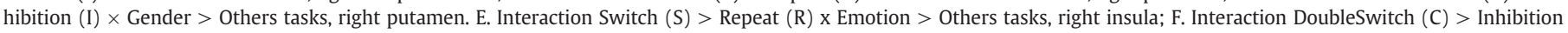
(I) $\times$ Emotion $>$ Others tasks, right insula; All regions are displayed on the mean structural image at $\mathrm{p}<0.001$ uncorrected.

slowing of reaction time due to the switch cost and the effect of inhibition ( $\mathrm{N}-2$ task-repetition cost), demonstrating that our paradigm successfully separated these two processes as proposed by previous work in cognitive psychology (Koch et al., 2010; Mayr and Keele, 2000). Our neuroimaging data revealed several regions (including the superior and medial SPL) commonly activated for switching in the different tasks, but with a reduction of these activations when switching to a previously inhibited task ( $\mathrm{N}-2$ task-repetition). No common increase across tasks was associated with inhibition or overcoming it. Rather, we show for the first time that inhibition leads to decreases in brain regions that are related to the specific demands of the inhibited task. In addition, we found that the emotional task produced more variable and less robust inhibition costs in behavioral performance, in parallel with an interaction between switching and inhibition effects in the anterior cingulate cortex. These novel data extend previous work on task switching in a number of ways, as discussed below.

\section{Neural substrates of task-switching}

Our fMRI results indicate that switching produces similar activations in medial and posterior parietal areas across all three different tasks. These findings accord with numerous studies showing parietal lobe activations for switching conditions in humans (Gajewski and Falkenstein, 2011; Wylie et al., 2003), as well as in primates recorded with single-unit electrophysiology (Kamigaki et al., 2009) or fMRI (Nakahara et al., 2002). Even though not totally similar, our results converge with those of Yeung et al. (2006) showing that some brain regions recruited during switching are specific to the task, while others are common to the switching process per se irrespective of the task. Here we found that both medial SPL and posterior IPS were activated by switching in all task conditions.

Parietal activations have long been associated with cognitive operations mediating attentional shifts in various domains, particularly between visual locations or features (Fink et al., 1997; Le et al., 1998), rather than with the task rule maintenance (Ravizza and Carter, 2008). However, posterior parietal activations are not restricted to attentional shifts based on spatial or visual information, but contribute to categorical perceptual decisions about sensory stimuli (Gurd et al., 2002). Some authors also suggested that parietal activation in task-switching and other executive tasks may be related to the selection of relevant stimulus-responses association (Derrfuss et al., 2004). In our case there was no difference in elementary stimulus-response mapping, but switching implied a change in selective attentional demand in order to extract the currently relevant stimulus features. 

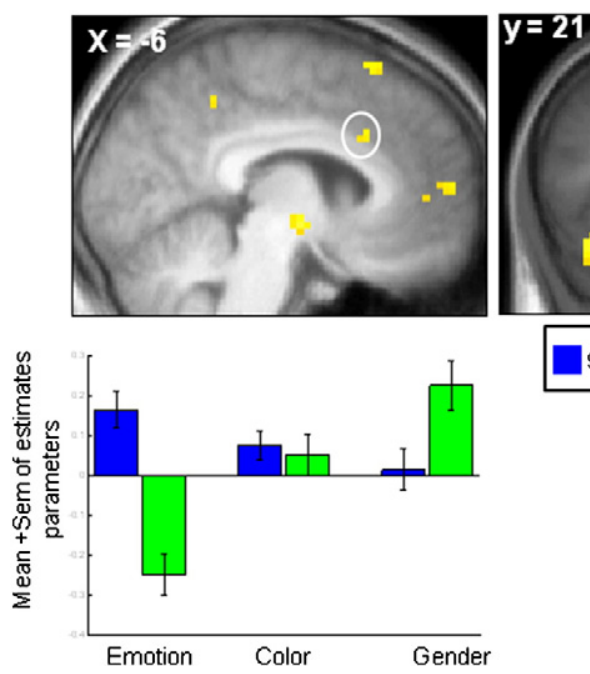

Fig. 5. Interaction of inhibition with emotion. Triple interaction testing for regions showing a selective reduction of activity on switching trials with an inhibition component in the emotion task $(\mathrm{C}>\mathrm{I})^{*}(\mathrm{~S}>\mathrm{R})^{*}$ (Emotion $>$ Others), highlighting a deactivation in ACC. Parameter estimates are plotted for the Switch and Inhibition conditions in each task.

In a previous study (Rushworth et al., 2001) that aimed at differentiating distinct processes in parietal cortex, the authors also related intentional switching of stimulus-response mapping rules to both IPS and posterior SPL. In this study, the medial SPL was activated by both visual and response switching tasks, with a peak of activation very close to the region found in our study. Our finding thus fits well with the idea that the superior-medial parietal cortex represents key neural substrates for switching abilities across different domains and modalities (Rushworth et al., 2001). Other studies have also found medial SPL activations in switching condition that were not task-specific (Kimberg et al., 2000; Wager et al., 2005), although these were reported under different anatomical labels (e.g., precuneus), but again with peak coordinates very close to the one found here. Likewise, in a study comparing face and word processing, Yeung et al. (2006) showed that, among a wide network of regions associated with task switching, the posterior medial parietal cortex was task-insensitive. Medial SPL was also commonly activated by different type of switch in a study comparing spatial attention and rule-response shifts (Chiu and Yantis, 2009). Finally, a recent meta-analysis reported that a conjunction map for three different kinds of switching (perceptual, response, and context) produced a selective overlap in the left posterior parietal cortex (MNI coordinates $\mathrm{x}$, $\mathrm{y}, \mathrm{z}=-25,-72,35)$ as well as the left inferior frontal cortex (Kim et al., 2012). Therefore, in agreement with previous work, we propose that the medial SPL, which activated to all switching conditions in our experiment, may play a crucial and general role in task reconfiguration processes that imply a change in current processing demands and thus require an extradimensional shift (Wager et al., 2004, 2005).

Activation in left intraparietal sulcus during task switching has also been reported in several imaging studies, again very close to our own peak coordinates (Dreher and Grafman, 2003; Ruge et al., 2005; Rushworth et al., 2001; e.g. Sohn et al., 2000). In a study aiming at differentiating inhibition and switching in executive control, a region specific to shifting was identified in a parietal area overlapping with IPS (Hedden and Gabrieli, 2011). However, because IPS appears engaged in both dual task and task switching conditions, some authors proposed that it might mediate a more general process converting sensory input into motor output, activated in a variety of tasks (Dreher and Grafman, 2003). IPS activation has also been reported for repeat trials in task switching paradigms (Dove et al., 2000; Le et al., 1998; Rushworth et al., 2001), in accord of the view that this region has a more general role in endogenous control of selective attention (Shulman et al., 2009).
Somewhat surprisingly, we did not find prefrontal cortex (PFC) increases on switching trials, although PFC activations are often related to executive control abilities including task switching and task set implementation. Some studies found reliable differences in dorsolateral frontal regions between switch and repeat conditions (DiGirolamo et al., 2001; Dove et al., 2000; Dreher and Berman, 2002; Sohn et al., 2000), whereas others found PFC activations without such differences between conditions (Dreher et al., 2002; Gurd et al., 2002; Kimberg et al., 2000). However, some studies failed to observe significant frontal effects at all but found only parietal and subcortical increases for the switch versus repeat condition (Barber and Carter, 2005; Forstmann et al., 2005). One possible explanation is that dorsolateral prefrontal cortex represents the abstract task set and hence is recruited for both switch and repeat trials (Wager et al., 2004; Wylie et al., 2004). Keeping multiple task sets at a relatively high level of activation (as here) has been shown to activate the anterior prefrontal cortex in a sustained manner (Sakai, 2008). In this case, activations would presumably not to be seen when contrasting the two conditions. Another explanation could be that in situations in which switch frequency is high and stimuli bivalent or trivalent (as here also), the participants actually expect a switch on each trial even when there is none (Altmann, 2004; Mayr and Kliegl, 2000), leading to a constant activation of switch-related regions in PFC. One solution to deal with this limitation could be to reduce the proportion of switch relative to repeat trials or to use a different baseline. However, we would rather favor the idea that even though the frontal cortex may contribute to maintaining a representation of the currently relevant task, the parietal cortex might be primarily responsible for disengaging, switching, and re-engaging "attention" between tasks (Collette et al., 2005; Wylie et al., 2003).

\section{Repetition and intradimensional switch}

Contrasting repeat versus switch trials revealed a fronto-striatoparietal network including right supramarginal gyrus, left caudate nucleus, and bilateral dorsolateral prefrontal areas. These regions might at first appear surprising given their role in executive functions and the more simple cognitive operations engaged during repeat trials. However different factors can explain these findings. First, as noted above, some studies observed similar prefrontal increases for both switch and repeat trials, when compared to baseline, leading to the conclusion that even repeat trials may comprise some switching component (Brass and von Cramon, 2002; Dove et al., 2000). Second, fronto-parietal activity in the repeat conditions could also be explained by the fact that even if the task rule does not change, the stimuli do, and the subject actually has to perform an "intradimensional" shift (Robbins, 2007) to pursue the same cognitive task on the new stimuli. For example, even when the cue "color" was repeated from a given trial to the next, and the position of the three faces was unchanged, the faces or their color could change (e.g. two green vs two red out of the three). This explanation seems all the more plausible given that, during informal debriefing, our participant reported that they did not experience repeat trials as subjectively much easier than switch trials. On the other hand, activation in caudate nucleus could represent an early phase of procedural or habit learning due to task repetition (Packard and Knowlton, 2002), as proposed for some components of response mapping and preparation across successive trials (Brass and von Cramon, 2002).

\section{Neural substrates of inhibition during task switching}

Our critical comparison of ABA versus CBA trials aimed at identifying the consequence of inhibition when switching from one task to another, on top of the switching process itself. Our results revealed that neural systems associated with a given task's demands were not only selectively recruited when switching to this task anew (on the simple switch 
condition), but also showed selective decreases in activity when returning to this task after it had been interrupted (and presumably inhibited) on the $\mathrm{N}-2$ trial (see Fig. 5). These data support the notion that task-related representations might be suppressed when switching away from a current task to engage with a new task, as proposed by psychological accounts of task switching (Koch et al., 2010; Mayr and Keele, 2000), and reveal for the first time the neural substrates of these effects in a face categorization task. However we do not replicate the results of Dreher and Berman (2002), who found increases in the right lateral prefrontal cortex and tempo-occipital areas when using a similar paradigm to study the overcoming of task inhibition. The right lateral PFC has been linked to inhibition in a variety of tasks (Aron, 2007; Cojan et al., 2009). However, the task employed by Dreher and Berman (2002) had a totally different timing, different stimuli, and probably different level of difficulty. Our task imposed a very quick response rate in order to obtain reliable behavioral N-2 task-repetition costs, as extensively verified in our pilot studies. Because the fine-tuned processes associated with inhibition may decay with time (Koch et al., 2004), differences in the inter-trial timing and distribution might explain why these authors found a modulation of PFC but we did not. Moreover, Dreher and Berman (2002) did not report the comparison between Switch and Repeat conditions (BBA versus AAA), and it is therefore possible that, due to the slower timing of their task, a similar effect in PFC would also arise in this contrast.

Moreover, in our study, we did not expect to observe activation in the right inferior frontal gyrus (rIFG) on inhibition trials (i.e. contrast $A B A$ versus $C B A$ ) since this comparison primarily aimed at determining the functional consequences (rather than the cause) of task inhibition - namely, which brain areas may show a reduced activation after being inhibited due to switching to a new task. Furthermore, the rIFG has been involved in more general executive control processes rather than inhibition alone (Hampshire et al., 2010). Consistent activations in rIFG were generally found in Go-NoGo or Wisconsin Cards Sorting tasks (Konishi et al., 1998, 1999, 2002), which may encompass inhibition processes distinct from those associated with pure task switching. Moreover, the concept of inhibition refers to different abilities (see Aron, 2007), including not only the suppression of a prepotent motor response (Sylvester et al., 2003) such as Go-NoGo (Swainson et al., 2003) or stop signal tasks (Aron et al., 2003), but also the resolution of interference by incongruent information (e.g. Friedman and Miyake, 2004; Hyafil et al., 2009). These processes are probably different from the backward inhibition that is necessary to facilitate shifting from one cognitive task to another.

Importantly, our study adapted the original task switching paradigm developed by Mayr and Keele (2000), where both switching and inhibition components (i.e. relative cost in response times) can be separately and reliably examined (in the same task) as a function of the N-1 and N-2 trial succession. Taken together, our fMRI data suggests that inhibition mechanisms operating in this context do not rely on a single neural system across different tasks, although they lead to a global decrease in posterior parietal areas on inhibition trials relative to pure switching trials, across all three tasks.

More critically, we found that on inhibition trials, each task was associated with reduced activity in brain regions that also exhibited specific task-related increases during switching. Thus, early occipital and posterior fusiform cortex showed selective decreases in the color task, whereas decreases were found in the insula, ventral striatum, and STS for the emotion task, and in more extensive regions in basal ganglia and prefrontal areas for the gender task. These effects are consistent with a role of occipito-temporal areas in color processing (Hadjikhani et al., 1998; Wandell and Winawer, 2011), and of both the insula and STS in emotion processing (Adolphs, 2003; Duerden et al., 2013; Said et al., 2011). The modulation of frontostriatal circuits during gender processing is less clear but might reflect a distinctive role of these regions in overlearned category-based discriminations. These effects suggest that brain areas distinctively recruited for specific task demands were less efficiently activated when switching to this task shortly after it was inhibited (on N-2 trials). Alternatively, we cannot rule out the possibility that activation of the striatum for both emotion and gender was merely a marker of task difficulty. However, other differences between tasks are unlikely to be explained by general effects of difficulty or attention, since it would imply that different brain areas were modulated by this general factor depending on task or that different kinds of attentional effects occurred in each task. Moreover, at least two of the tasks had similar RTs, yet showed different patterns in brain activity, indicating that difficulty per se is unlikely to cause these effects. Finally, it is remarkable that the same distribution of activation was obtained for each task by performing two distinct contrasts (see Fig. 4), based on totally different trials (Switch $>$ Repeat and DoubleSwitch $>$ Inhibition for each task separately). This internal replication supports our interpretation that neural processes whose activity was reduced following inhibition of the former task demands (highlighted by the contrast DoubleSwitch $>$ Inhibition) corresponded to those processes that are necessary when switching to a new task (highlighted by the contrast Switch $>$ Repeat). Future studies might further explore these overlaps by using pre-defined functional localizers to better probe activity in brain networks engaged in each task.

In any case, to our knowledge, these data provide the first neural evidence for an inhibition of task-specific processes during taskswitching (on trial $\mathrm{N}-2$ ), thus accounting for a less efficient engagement of the same processes on subsequent return to the same task (on trial $\mathrm{N}$ ). These new findings therefore strongly validate the existence of inhibitory process as part of the switch cost, a phenomenon that has hitherto remained debated in the literature (Kiesel et al., 2010; Koch et al., 2010).

\section{Emotion modulates switching and inhibition}

A secondary goal of our study was to determine any distinctive impact of emotional information on cognitive control processes mediating task switching and inhibition, as previously reported for other cognitive and attentional functions (Compton, 2003; Sagaspe et al., 2011). At the neural level, comparing conditions with a switching component alone (Switch trials) with those involving switching plus the effect of inhibition (Inhibition trials) for the emotional task, relative to the same comparison for the other (gender and color) tasks, allowed us to identify any differential modulation associated with the different costs in each task. This analysis revealed a specific decrease in ACC activity for inhibition trials during the emotional task, but not during the two other tasks. Thus, ACC was less strongly recruited when returning to the emotion task after this task-set was curtailed on $\mathrm{N}-2$ trials, relative to returning to another task, suggesting weaker effects of inhibition in the former than the latter conditions. Accordingly, though substantial on average, the behavioral inhibition costs were found to be less robust in the emotion than in the other tasks.

ACC has been associated with conflict monitoring and error processing (Chechko et al., 2012; Egner et al., 2008; Nee et al., 2011; Roberts and Hall, 2008) as well as resistance to external interference (Nee et al., 2007). In our paradigm, it seems plausible that ACC was generally activated when switching to a new task, particularly when this demanded higher attentional control and effort (Rushworth et al., 2007) to avoid any carryover interference and/or to overcome the inhibition of the preceding task. This was true for both the Switch and DoubleSwitch trials across all task conditions. However, we found that the emotional task produced a relative and marked deactivation of ACC on Inhibition trials, compatible with the idea that emotion processing did not suffer the same degree of inhibition as observed in the gender and color tasks. This resistance to inhibition during task switching might reflect a specific advantage and relative "automaticity" in emotion processing, as compared with the more cognitive demands of the other tasks. Emotion is known to facilitate perception, attention, and memory in various situations (Vuilleumier, 2005), and social emotional processing tends to 
operate swiftly without explicit attention and without effort (Lieberman, 2007). Our study shows for the first time that emotion also modulates the effect of inhibition and switching in task-set reconfiguration, with a corresponding impact on the recruitment of ACC during switching.

It is worth noting that inhibition in task-switching has recently been investigated in neuropsychiatric diseases like obsessive compulsive disorder or Parkinson's disease (Fales et al., 2006; Moritz et al., 2004), although results still remain partly inconclusive. Because of the particular effect of emotional information on task-switching observed here, we suggest that such emotion effects should be more systematically tested in neuropsychiatry pathologies that often involve impairments in both emotion regulation and cognitive flexibility (Meiran et al., 2011) - e.g. like depression. In particular, impaired inhibition in task-switching is a marker of cognitive inflexibility and has been linked to repetitive thoughts (Whitmer and Banich, 2007). De Lissnyder et al. (2010) also reported that while the severity of depressive symptoms does not correlate with impairment in switching or inhibition, people with a high tendency to ruminate tend to present reduced inhibition ability, especially for negative material. Further research on cognitive and neural mechanisms underlying the interaction between emotion and task switching process might therefore yield useful insights on clinical conditions associated with thought disorders. Additional measures related to personality traits such as anxiety or mood states might also help elucidate the source of individual variability in executive control in the presence of emotional stimuli (e.g. see Murphy et al., 2012).

\section{Conclusion}

Using a novel task switching paradigm, we demonstrate a key role of the left medial SPL in switching processes irrespective of task demands, with concomitant recruitment of other parietal regions in IPS and PCC whose exact role still awaits clarification by future studies. Inhibition of a previous task set during switching was found to produce a relative deactivation of these parietal regions when returning to the same task, together with task-specific reductions in other regions concerned with specific task demands. These findings support the existence of inhibition processes during task-switching, and provide a neural substrate for the inhibition cost (in RTs) observed behaviorally in such paradigms. In addition, emotional information appeared to have a differential impact on the inhibition component of task switching, leading to reduced recruitment of ACC when returning to a previously inhibited task-set relative to other task conditions, and greater variability across individuals. This interaction between affect and switching processes might be relevant to better understand cognitive inflexibility and thought control disorders in some psychiatric disorders, an issue that needs to be further investigated in clinical populations.

\section{Role of funding source}

$\mathrm{CP}, \mathrm{VS}, \mathrm{YC}$ and PV are supported by the Swiss National Science Foundation. PV is additionally supported by the FOREMANE fund (Société académique). CP and MD received a grant from the Vachoux Foundation. MD is also supported by the Belgian National Science Foundation.

\section{Acknowledgments}

We thank Sandra Chanraud for her comments on a previous version of the manuscript.

\section{Conflict of interest}

None declared

\section{References}

Adolphs, R., 2003. Cognitive neuroscience of human social behaviour. Nat. Rev. Neurosci. 4, 165-178.

Allport, A., Wylie, G., 2000. Task-switching, stimulus-response bindings, and negative priming. In: Monsell, S., Driver, J. (Eds.), Control of Cognitive Processes, Attention and Performance XVIII. MIT Press, London.

Altmann, E.M., 2004. Advance preparation in task switching: what work is being done? Psychol. Sci. 15, 616-622.

Arbuthnott, K.D., 2008. Asymmetric switch cost and backward inhibition: carryover activation and inhibition in switching between tasks of unequal difficulty. Can. J. Exp. Psychol. 62, 91-100.

Armony, J.L., Servan-Schreiber, D., Cohen, J.D., Ledoux, J.E., 1997. Computational modeling of emotion: explorations through the anatomy and physiology of fear conditioning. Trends Cogn. Sci. 1, 28-34.

Aron, A.R., 2007. The neural basis of inhibition in cognitive control. Neuroscientist 13 214-228.

Aron, A.R., Fletcher, P.C., Bullmore, E.T., Sahakian, B.J., Robbins, T.W., 2003. Stop-signal inhibition disrupted by damage to right inferior frontal gyrus in humans. Nat. Neurosci. 6, 115-116.

Aron, A.R., Robbins, T.W., Poldrack, R.A., 2004. Inhibition and the right inferior frontal cortex. Trends Cogn. Sci. 8, 170-177.

Barber, A.D., Carter, C.S., 2005. Cognitive control involved in overcoming prepotent response tendencies and switching between tasks. Cereb Cortex 15, 899-912.

Beck, A.T., Epstein, N., Brown, G., Steer, R.A., 1988. An inventory for measuring clinical anxiety: psychometric properties. J. Consult. Clin. Psychol. 56, 893-897.

Beck, A.T., Steer, R.A., Brown, G.K., 1996. Manual for the Beck Depression Inventory, 2nd edition. The Psychological Corporation, San Antonio.

Brass, M., von Cramon, D.Y., 2002. The role of the frontal cortex in task preparation. Cereb. Cortex 12, 908-914.

Brass, M., von Cramon, D.Y., 2004. Decomposing components of task preparation with functional magnetic resonance imaging. J. Cogn. Neurosci. 16, 609-620.

Chechko, N., Kellermann, T., Zvyagintsev, M., Augustin, M., Schneider, F., Habel, U. 2012. Brain circuitries involved in semantic interference by demands of emotional and non-emotional distractors. PLoS One 7, e38155.

Chiu, Y.C., Yantis, S., 2009. A domain-independent source of cognitive control for task sets: shifting spatial attention and switching categorization rules. J. Neurosci. 29, 3930-3938.

Cohen, N., Henik, A., Mor, N., 2011. Can emotion modulate attention? Evidence for reciprocal links in the attentional network test. Exp. Psychol. 58, 171-179.

Cojan, Y., Waber, L., Carruzzo, A., Vuilleumier, P., 2009. Motor inhibition in hysterical conversion paralysis. Neurolmage 47, 1026-1037.

Collette, F., Van der Linden, M., Laureys, S., Delfiore, G., Degueldre, C., Luxen, A., Salmon, E., 2005. Exploring the unity and diversity of the neural substrates of executive functioning. Hum. Brain Mapp. 25, 409-423.

Compton, R.J., 2003. The interface between emotion and attention: a review of evidence from psychology and neuroscience. Behav. Cogn. Neurosci. Rev. 2, 115-129.

Compton, R.J., Banich, M.T., Mohanty, A., Milham, M.P., Herrington, J., Miller, G.A., Scalf P.E., Webb, A., Heller, W., 2003. Paying attention to emotion: an fMRI investigation of cognitive and emotional Stroop tasks. Cogn. Affect Behav. Neurosci. 3, 81-96.

Corbetta, M., Patel, G., Shulman, G.L., 2008. The reorienting system of the human brain: from environment to theory of mind. Neuron 58, 306-324.

Crone, E.A., Wendelken, C., Donohue, S.E., Bunge, S.A., 2006. Neural evidence for dissociable components of task-switching. Cereb. Cortex 16, 475-486.

De Lissnyder, E., Koster, E.H., Derakshan, N., De Raedt, R., 2010. The association between depressive symptoms and executive control impairment in response to emotional and non-emotional information. Cogn. Emot. 24, 264-280.

Derrfuss, J., Brass, M., von Cramon, D.Y., 2004. Cognitive control in the posterior frontolateral cortex: evidence from common activations in task coordination, interference control, and working memory. Neurolmage 23, 604-612.

Derrfuss, J., Brass, M., Neumann, J., von Cramon, D.Y., 2005. Involvement of the inferior frontal junction in cognitive control: meta-analyses of switching and Stroop studies. Hum. Brain Mapp. 25, 22-34.

DiGirolamo, G.J., Kramer, A.F., Barad, V., Cepeda, N.J., Weissman, D.H., Milham, M.P. Wszalek, T.M., Cohen, N.J., Banich, M.T., Webb, A., Belopolsky, A.V., McAuley, E. 2001. General and task-specific frontal lobe recruitment in older adults during executive processes: a fMRI investigation of task-switching. Neuroreport 12, 2065-2071.

Dosenbach, N.U., Visscher, K.M., Palmer, E.D., Miezin, F.M., Wenger, K.K., Kang, H.C. Burgund, E.D., Grimes, A.L., Schlaggar, B.L., Petersen, S.E., 2006. A core system for the implementation of task sets. Neuron 50, 799-812.

Dove, A., Pollmann, S., Schubert, T., Wiggins, C.J., von Cramon, D.Y., 2000. Prefrontal cortex activation in task switching: an event-related fMRI study. Brain Res. Cogn. Brain Res. 9, 103-109.

Dreher, J.C., Berman, K.F., 2002. Fractionating the neural substrate of cognitive control processes. Proc. Natl. Acad. Sci. U. S. A. 99, 14595-14600.

Dreher, J.C., Grafman, J., 2003. Dissociating the roles of the rostral anterior cingulate and the lateral prefrontal cortices in performing two tasks simultaneously or successively. Cereb. Cortex 13, 329-339.

Dreher, J.C., Koechlin, E., Ali, S.O., Grafman, J., 2002. The roles of timing and task order during task switching. Neurolmage 17, 95-109.

Duerden, E.G., Arsalidou, M., Lee, M., Taylor, M.J., 2013. Lateralization of affective processing in the insula. NeuroImage 78C, 159-175.

Egner, T., Etkin, A., Gale, S., Hirsch, J., 2008. Dissociable neural systems resolve conflict from emotional versus nonemotional distracters. Cereb. Cortex 18, 1475-1484.

Ethofer, T., Gschwind, M., Vuilleumier, P., 2011. Processing social aspects of human gaze: a combined fMRI-DTI study. NeuroImage 55, 411-419. 
Fales, C.L., Vanek, Z.F., Knowlton, B.J., 2006. Backward inhibition in Parkinson's disease. Neuropsychologia 44, 1041-1049.

Fink, G.R., Halligan, P.W., Marshall, J.C., Frith, C.D., Frackowiak, R.S., Dolan, R.J., 1997 Neural mechanisms involved in the processing of global and local aspects of hierarchically organized visual stimuli. Brain 120 (Pt 10), 1779-1791.

Forstmann, B.U., Brass, M., Koch, I., von Cramon, D.Y., 2005. Internally generated and directly cued task sets: an investigation with fMRI. Neuropsychologia 43, 943-952.

Forstmann, B.U., Brass, M., Koch, I., von Cramon, D.Y., 2006. Voluntary selection of task sets revealed by functional magnetic resonance imaging. J. Cogn. Neurosci. 18, 388-398.

Friedman, N.P., Miyake, A., 2004. The relations among inhibition and interference control functions: a latent-variable analysis. J. Exp. Psychol. Gen. 133, 101-135.

Gajewski, P.D., Falkenstein, M., 2011. Diversity of the P3 in the task-switching paradigm. Brain Res. 1411, 87-97.

Gray, J.R., 2001. Emotional modulation of cognitive control: approach-withdrawal states double-dissociate spatial from verbal two-back task performance. J. Exp. Psychol. Gen. 130, 436-452.

Gray, J.R., Braver, T.S., Raichle, M.E., 2002. Integration of emotion and cognition in the lateral prefrontal cortex. Proc. Natl. Acad. Sci. U. S. A. 99, 4115-4120.

Gurd, J.M., Amunts, K., Weiss, P.H., Zafiris, O., Zilles, K., Marshall, J.C., Fink, G.R., 2002. Posterior parietal cortex is implicated in continuous switching between verbal fluency tasks: an fMRI study with clinical implications. Brain 125, 1024-1038.

Hadjikhani, N., Liu, A.K., Dale, A.M., Cavanagh, P., Tootell, R.B., 1998. Retinotopy and color sensitivity in human visual cortical area V8. Nat. Neurosci. 1, 235-241.

Hampshire, A., Chamberlain, S.R., Monti, M.M., Duncan, J., Owen, A.M., 2010. The role of the right inferior frontal gyrus: inhibition and attentional control. NeuroImage 50 1313-1319.

Hare, T.A., Tottenham, N., Galvan, A., Voss, H.U., Glover, G.H., Casey, B.J., 2008. Biological substrates of emotional reactivity and regulation in adolescence during an emotional go-nogo task. Biol. Psychiatry 63, 927-934.

Hedden, T., Gabrieli, J.D., 2011. Shared and selective neural correlates of inhibition, facilitation, and shifting processes during executive control. Neurolmage 51, 421-431.

Hubner, M., Dreisbach, G., Haider, H., Kluwe, R.H., 2003. Backward inhibition as a means of sequential task-set control: evidence for reduction of task competition. J. Exp. Psychol. Learn. Mem. Cogn. 29, 289-297.

Hyafil, A., Summerfield, C., Koechlin, E., 2009. Two mechanisms for task switching in the prefrontal cortex. J. Neurosci. 29, 5135-5142.

Kamigaki, T., Fukushima, T., Miyashita, Y., 2009. Cognitive set reconfiguration signaled by macaque posterior parietal neurons. Neuron 61, 941-951.

Kiesel, A., Steinhauser, M., Wendt, M., Falkenstein, M., Jost, K., Philipp, A.M., Koch, I., 2010. Control and interference in task switching-a review. Psychol. Bull. 136, 849-874.

Kim, C., Cilles, S.E., Johnson, N.F., Gold, B.T., 2012. Domain general and domain preferential brain regions associated with different types of task switching: a metaanalysis. Hum. Brain Mapp. 33, 130-142.

Kimberg, D.Y., Aguirre, G.K., D'Esposito, M., 2000. Modulation of task-related neural activity in task-switching: an fMRI study. Brain Res. Cogn. Brain Res. 10, 189-196.

Koch, I., Gade, M., Philipp, A.M., 2004. Inhibition of response mode in task switching. Exp. Psychol. 51, 52-58.

Koch, I., Gade, M., Schuch, S., Philipp, A.M., 2010. The role of inhibition in task switching: a review. Psychon. Bull. Rev. 17, 1-14.

Konishi, S., Nakajima, K., Uchida, I., Sekihara, K., Miyashita, Y., 1998. No-go dominant brain activity in human inferior prefrontal cortex revealed by functional magnetic resonance imaging. Eur. J. Neurosci. 10, 1209-1213.

Konishi, S., Nakajima, K., Uchida, I., Kikyo, H., Kameyama, M., Miyashita, Y., 1999. Common inhibitory mechanism in human inferior prefrontal cortex revealed by eventrelated functional MRI. Brain 122 (Pt 5), 981-991.

Konishi, S., Hayashi, T., Uchida, I., Kikyo, H., Takahashi, E., Miyashita, Y., 2002. Hemispheric asymmetry in human lateral prefrontal cortex during cognitive set shifting. Proc. Natl. Acad. Sci. U. S. A. 99, 7803-7808.

Le, T.H., Pardo, J.V., Hu, X., 1998. 4T-fMRI study of nonspatial shifting of selective attention: cerebellar and parietal contributions. J. Neurophysiol. 79, 1535-1548.

Lieberman, M.D., 2007. Social cognitive neuroscience: a review of core processes. Annu. Rev. Psychol. 58, 259-289.

Lieberman, M.D., Cunningham, W.A., 2009. Type I and Type II error concerns in fMRI research: re-balancing the scale. Soc. Cogn. Affect Neurosci. 4, 423-428.

Lien, M.C., Ruthruff, E., Kuhns, D., 2006. On the difficulty of task switching: assessing the role of task-set inhibition. Psychon. Bull. Rev. 13, 530-535.

Luks, T.L., Simpson, G.V., Feiwell, R.J., Miller, W.L., 2002. Evidence for anterior cingulate cortex involvement in monitoring preparatory attentional set. Neurolmage 17, 792-802.

Lundqvist, D., Flykt, A., Ohman, A., 1998. The Karolinska Directed Emotional Faces (CD-ROM). Departement of Clinical Neuroscience, Psychology Section, Karolinska Institut, Stockholm, Sweden.

Mayr, U., 2002. Inhibition of action rules. Psychon. Bull. Rev. 9, 93-99.

Mayr, U., Keele, S.W., 2000. Changing internal constraints on action: the role of backward inhibition. J Exp Psychol Gen 129, 4-26.

Mayr, U., Kliegl, R., 2000. Task-set switching and long-term memory retrieval. J. Exp. Psychol. Learn. Mem. Cogn. 26, 1124-1140.

Mayr, U., Diedrichsen, J., Ivry, R., Keele, S.W., 2006. Dissociating task-set selection from task-set inhibition in the prefrontal cortex. J. Cogn. Neurosci. 18, 14-21.

Meiran, N., 2010. Task-Switching. In: Hassin, R. (Ed.), Self Control in Society, Mind, and Brain. Oxford University Press, New York.

Meiran, N., Diamond, G.M., Toder, D., Nemets, B., 2011. Cognitive rigidity in unipolar depression and obsessive compulsive disorder: examination of task switching
Stroop, working memory updating and post-conflict adaptation. Psychiatry Res $185,149-156$.

Monsell, S., 2003. Task switching. Trends Cogn. Sci. 7, 134-140.

Moritz, S., Hubner, M., Kluwe, R., 2004. Task switching and backward inhibition in obsessive-compulsive disorder. J. Clin. Exp. Neuropsychol. 26, 677-683.

Murphy, F.C., Michael, A., Sahakian, B.J., 2012. Emotion modulates cognitive flexibility in patients with major depression. Psychol. Med. 42, 1373-1382.

Nakahara, K., Hayashi, T., Konishi, S., Miyashita, Y., 2002. Functional MRI of macaque monkeys performing a cognitive set-shifting task. Science 295, 1532-1536.

Nee, D.E., Wager, T.D., Jonides, J., 2007. Interference resolution: insights from a metaanalysis of neuroimaging tasks. Cogn. Affect Behav. Neurosci. 7, 1-17.

Nee, D.E., Kastner, S., Brown, J.W., 2011. Functional heterogeneity of conflict, error, task-switching, and unexpectedness effects within medial prefrontal cortex. NeuroImage 54, 528-540.

Oldfield, R.C., 1971. The assessment and analysis of handedness: the Edinburgh inventory. Neuropsychologia 9, 97-113.

Packard, M.G., Knowlton, B.J., 2002. Learning and memory functions of the Basal Ganglia. Annu. Rev. Neurosci. 25, 563-593.

Philipp, A.M., Kalinich, C., Koch, I., Schubotz, R.I., 2008. Mixing costs and switch costs when switching stimulus dimensions in serial predictions. Psychol. Res. 72, 405-414.

Pollmann, S., Dove, A., Yves von Cramon, D., Wiggins, C.J., 2000. Event-related fMRI: comparison of conditions with varying BOLD overlap. Hum. Brain Mapp. 9, 26-37.

Ravizza, S.M., Carter, C.S., 2008. Shifting set about task switching: behavioral and neural evidence for distinct forms of cognitive flexibility. Neuropsychologia 46, 2924-2935.

Ravizza, S.M., Ciranni, M.A., 2002. Contributions of the prefrontal cortex and basal ganglia to set shifting. J. Cogn. Neurosci. 14, 472-483.

Robbins, T.W., 2007. Shifting and stopping: fronto-striatal substrates, neurochemical modulation and clinical implications. Philos. Trans. R. Soc. Lond. B. Biol. Sci. 362, 917-932.

Roberts, K.L., Hall, D.A., 2008. Examining a supramodal network for conflict processing: a systematic review and novel functional magnetic resonance imaging data for related visual and auditory Stroop tasks. J. Cogn. Neurosci. 20, 1063-1078.

Ruge, H., Brass, M., Koch, I., Rubin, O., Meiran, N., von Cramon, D.Y., 2005. Advance preparation and stimulus-induced interference in cued task switching: further insights from BOLD fMRI. Neuropsychologia 43, 340-355.

Rushworth, M.F., Paus, T., Sipila, P.K., 2001. Attention systems and the organization of the human parietal cortex. J. Neurosci. 21, 5262-5271.

Rushworth, M.F., Hadland, K.A., Paus, T., Sipila, P.K., 2002. Role of the human medial frontal cortex in task switching: a combined fMRI and TMS study. J. Neurophysiol. $87,2577-2592$

Rushworth, M.F.S., Buckley, M.J., Behrens, T.E.J., Walton, M.E., Bannerman, D.M., 2007. Functional organization of the medial frontal cortex. Curr. Opin. Neurobiol. 17, 220-227.

Sagaspe, P., Schwartz, S., Vuilleumier, P., 2011. Fear and stop: a role for the amygdala in motor inhibition by emotional signals. Neurolmage 55, 1825-1835.

Said, C.P., Haxby, J.V., Todorov, A., 2011. Brain systems for assessing the affective value of faces. Phil. Trans. R. Soc. B 366, 1660-1670.

Sakai, K., 2008. Task set and prefrontal cortex. Annu. Rev. Neurosci. 31, 219-245.

Shulman, G.L., Astafiev, S.V., Franke, D., Pope, D.L.W., Snyder, A.Z., McAvoy, M.P., Corbetta, M., 2009. Interaction of stimulus-driven reorienting and expectation in ventral and dorsal frontoparietal and basal ganglia-cortical networks. J. Neurosci. 29, 4392-4407.

Sohn, M.H., Ursu, S., Anderson, J.R., Stenger, V.A., Carter, C.S., 2000. The role of prefrontal cortex and posterior parietal cortex in task switching. Proc. Natl. Acad. Sci. U. S. A. 97, $13448-13453$.

Swainson, R., Cunnington, R., Jackson, G.M., Rorden, C., Peters, A.M., Morris, P.G., Jackson, S.R., 2003. Cognitive control mechanisms revealed by ERP and fMRI: evidence from repeated task-switching. J. Cogn. Neurosci. 15, 785-799.

Sylvester, C.Y., Wager, T.D., Lacey, S.C., Hernandez, L., Nichols, T.E., Smith, E.E., Jonides, J., 2003. Switching attention and resolving interference: fMRI measures of executive functions. Neuropsychologia 41, 357-370.

Vuilleumier, P., 2005. How brains beware: neural mechanisms of emotional attention. Trends Cogn. Sci. 9, 585-594 (\%U http://www.sciencedirect.com/science/article/ B6VH9-4HHWWHM-1/2/027fdf51d53110a9d541b427a5701182).

Vuilleumier, P., 2009. The Role of the Human Amygdala in Perception and Attention. In: Whalen, P.J., Phelps, E.A. (Eds.), The Human Amygdala. Guilford Press.

Wager, T.D., Jonides, J., Reading, S., 2004. Neuroimaging studies of shifting attention: a meta-analysis. NeuroImage 22, 1679-1693.

Wager, T.D., Jonides, J., Smith, E.E., Nichols, T.E., 2005. Toward a taxonomy of attention shifting: individual differences in fMRI during multiple shift types. Cogn. Affect Behav. Neurosci. 5, 127-143.

Wandell, B.A., Winawer, J., 2011. Imaging retinotopic maps in the human brain. Vis. Res. 51, 718-737.

Whitmer, A.J., Banich, M.T., 2007. Inhibition versus switching deficits in different forms of rumination. Psychol. Sci. 18, 546-553.

Woodward, T.S., Ruff, C.C., Ngan, E.T., 2006. Short- and long-term changes in anterior cingulate activation during resolution of task-set competition. Brain Res 1068, 161-169.

Wylie, G.R., Javitt, D.C., Foxe, J.J., 2003. Task switching: a high-density electrical mapping study. NeuroImage 20, 2322-2342.

Wylie, G.R., Javitt, D.C., Foxe, J.J., 2004. Don't think of a white bear: an fMRI investigation of the effects of sequential instructional sets on cortical activity in a taskswitching paradigm. Hum Brain Mapp 21, 279-297.

Yeung, N., Nystrom, L.E., Aronson, J.A., Cohen, J.D., 2006. Between-task competition and cognitive control in task switching. J. Neurosci. 26, 1429-1438. 